\title{
Controlled ovarian stimulation and progesterone supplementation affect vaginal and endometrial microbiota in IVF cycles: a pilot study
}

\author{
Andrea Carosso ${ }^{1}$ D $\cdot$ Alberto Revelli $^{1}$ - Gianluca Gennarelli ${ }^{1} \cdot$ Stefano Canosa $^{1}$ - Stefano Cosma ${ }^{1} \cdot$ Fulvio Borella $^{1}$. \\ Annalisa Tancredi ${ }^{1}$ - Carlotta Paschero ${ }^{1}$ - Lara Boatti ${ }^{2}$. Elisa Zanotto ${ }^{3}$. Francesca Sidoti ${ }^{3}$. Paolo Bottino ${ }^{3}$. \\ Cristina Costa $^{3} \cdot$ Rossana Cavallo $^{3} \cdot$ Chiara Benedetto $^{1}$
}

Received: 25 March 2020 / Accepted: 30 June 2020 / Published online: 15 July 2020

(C) The Author(s) 2020

\begin{abstract}
Purpose Does controlled ovarian stimulation (COS) and progesterone (P) luteal supplementation modify the vaginal and endometrial microbiota of women undergoing in vitro fertilization?

Methods Fifteen women underwent microbiota analysis at two time points: during a mock transfer performed in the luteal phase of the cycle preceding COS, and at the time of fresh embryo transfer (ET). A vaginal swab and the distal extremity of the ET catheter tip were analyzed using next-generation 16SrRNA gene sequencing. Heterogeneity of the bacterial microbiota was assessed according to both the Bray-Curtis similarity index and the Shannon diversity index.

Results Lactobacillus was the most prevalent genus in the vaginal samples, although its relative proportion was reduced by COS plus P supplementation $(71.5 \pm 40.6 \%$ vs. $61.1 \pm 44.2 \%)$. In the vagina, an increase in pathogenic species was observed, involving Prevotella $(3.5 \pm 8.9 \%$ vs. $12.0 \pm 19.4 \%)$, and Escherichia coli-Shigella spp. $(1.4 \pm 5.6 \%$ vs. $2.0 \pm 7.8 \%)$. In the endometrium, the proportion of Lactobacilli slightly decreased (27.4 $\pm 34.5 \%$ vs. $25.0 \pm 29.9 \%$ ); differently, both Prevotella and Atopobium increased $(3.4 \pm 9.5 \%$ vs. $4.7 \pm 7.4 \%$ and $0.7 \pm 1.5 \%$ vs. $5.8 \pm 12.0 \%)$. In both sites, biodiversity was greater after $\operatorname{COS}(p<0.05)$, particularly in the endometrial microbiota, as confirmed by Bray-Curtis analysis of the phylogenetic distance among bacteria genera. Bray-Curtis analysis confirmed significant differences also for the paired endometrium-vagina samples at each time point.

Conclusions Our findings suggest that COS and P supplementation significantly change the composition of vaginal and endometrial microbiota. The greater instability could affect both endometrial receptivity and placentation. If our findings are confirmed, they may provide a further reason to encourage the freeze-all strategy.
\end{abstract}

Keywords Microbiota - Vagina · Endometrium · IVF/IVF-ICSI · Controlled ovarian stimulation · Dysbiosis · Infertility · Reproductive tract bacteria $\cdot$ Embryo implantation $\cdot 16 \mathrm{~S}$ ribosomal subunit $\cdot$ Freeze all

\section{Introduction}

The microbiota of the female reproductive tract has long been studied through cultivation methods to identify the

Andrea Carosso

andrea88.carosso@gmail.com

1 Obstetrics and Gynecology 1U, Physiopathology of Reproduction and IVF Unit, Department of Surgical Sciences, Sant Anna Hospital, University of Torino, Via Ventimiglia 1, 10126 Turin, Italy

2 Arrow Diagnostics S.r.1., Via Francesco Rolla 26, 16152 Genoa, Italy

3 Virology, Public Health and Pediatrics Department, University of Torino, Turin, Italy microorganisms that can be isolated and to assess their impact on reproductive physiology. However, an accurate picture of the microbial diversity in this body niche was achieved only recently, following the advent of highly sensitive molecular techniques that can identify microorganisms that cannot be grown in culture [1]. Sequencing of the bacterial $16 \mathrm{~S}$ ribosomal RNA (rRNA) gene, encoding an essential component of the ribosome, is one of the most effective techniques in this field. The rRNA gene has hypervariable regions (V1-V9) that can be used to distinguish among even extremely similar, otherwise undistinguishable, bacteria [2].

Next-generation sequencing (NGS) methods such as $16 \mathrm{~S}$ rRNA gene sequencing can be used to better define the bacterial community physiologically residing in the female reproductive tract: mainly Lactobacilli but also a small proportion 
of Prevotella, Gardnerella, Atopobium, Sneathia, Bifidobacterium, Megasphaera, and Anaerococcus [3-5].

Bacteria in the vagina, endometrium, and follicular fluid are likely to affect the reproductive process, from fertilization to implantation, and from maintenance of pregnancy to microbial colonization of the newborn $[6,7]$. With regard to in vitro fertilization (IVF), the presence of certain bacterial strains in the endometrium was reported to reduce the likelihood of embryo implantation and, ultimately, the pregnancy rate $[8$, 9].

The physiological variability of ovarian steroid circulating levels during the normal menstrual cycle was shown to induce changes in vaginal microbiota [10]. It seems likely that the progressive, supraphysiological increase in estradiol (E2) serum levels during controlled ovarian stimulation (COS), as well as the iatrogenic increase in progesterone $(\mathrm{P})$ concentration resulting from luteal phase $\mathrm{P}$ supplementation, induces significant changes in the vaginal and possibly also in the endometrial microbiota. How these changes influence endometrial receptivity toward embryo implantation is still largely unknown. An estrogen (or progesterone)-induced alteration of the vaginal and the endometrial microbiota could add a microbiological perspective to explain why implantation rates are reportedly higher in frozen embryo-transfer (ET) cycles vs. fresh ET following COS [11-13].

The aim of this pilot study was to investigate whether COS and progesterone luteal phase supplementation in IVF patients induce changes in the vaginal and the endometrial microbiota that could potentially influence endometrial receptivity toward embryo implantation. The changes were evaluated by using both the Bray-Curtis similarity index and the Shannon index to compare microbiota heterogeneity and stability in the two sites before and after COS. Furthermore, variation in the concentration of the individual bacterial genera was assessed.

\section{Materials and methods}

\section{Patients}

The study was authorized by the local ethical committee (authorization no. 0092218).

Fifteen patients undergoing IVF at the University Reproductive Physiopathology Center of Sant' Anna Hospital, Turin were selected to participate in the study. Inclusion criteria were Caucasian ethnicity, age $\leq 42$ years, basal (day 3) follicle-stimulating hormone (FSH) $<15 \mathrm{IU} /$, anti-müllerian hormone $(\mathrm{AMH})>0.2 \mathrm{ng} / \mathrm{ml}$, regular menstrual cycles (25-32 days), no previous repeated implantation failure ( $\geq 3$ ETs of good scored embryos, with no pregnancy) or recurrent miscarriage ( $>3$ previous first trimester miscarriages), absence of tubal or uterine pathology (e.g., hydrosalpinx, endometrial polyps, myomas), pPROM history, and gastrointestinal disease. Patients receiving antibiotic or probiotic therapy possibly interfering with endometrial and vaginal microbiota were also excluded. Patients with a recent history of cervico-vaginal infection were also excluded; cervico-vaginal swabs were taken in all patients within 6 months prior to treatment. The swabs were analyzed by sowing on CNA agar plates (nalidixic acid colistin) followed by the MALDI-TOF technique for the identification of the most common cervico-vaginal pathogens plus real-time PCR for Chlamydia trachomatis, Neisseria gonorrhoeae, Trichomonas vaginalis, Mycoplasma genitalium, Mycoplasma hominis, Ureaplasma parvum, and Ureaplasma urealiticum.

\section{Controlled ovarian stimulation and IVF}

COS was accomplished using recombinant FSH (rFSH; Gonal F®; Merck, Germany); the starting dose ranged between 100 and $300 \mathrm{IU} / 1$ and was tailored according to antral follicle count (AFC), AMH, and body mass index (BMI, weight in $\mathrm{kg}$ /height in $\mathrm{m}^{2}$ ). The initial $\mathrm{rFSH}$ daily dose was eventually adjusted at the initial assessment of ovarian response (day 6-7 of stimulation). Pituitary suppression was achieved by administration of GnRH-antagonist cetrorelix (Cetrotide ${ }^{\circledR}$, Merck, Germany) according to a fixed protocol, starting from day 6 of ovarian stimulation. COS was monitored by serial transvaginal ultrasound (TV-US) plus serum estradiol (E2) measurement starting on day 6-7 of COS, and then every second day until at least two dominant follicles reached $18 \mathrm{~mm}$ in diameter, with appropriate E2 levels. The cycle was interrupted when no more than one follicle $>10 \mathrm{~mm}$ in mean diameter was seen at US on day 6-7 and serum E2 was $<100 \mathrm{pg} / \mathrm{ml}$. Final follicular maturation was triggered by injecting subcutaneously 10,000 IU hCG (Gonasi HP®, IBSA, Switzerland); TV-US-guided oocyte pick-up (OPU) was performed approximately $36-37 \mathrm{~h}$ later under local anesthesia (paracervical block). Classical IVF or intracytoplasmic sperm injection (ICSI) was performed according to clinical indications. After 2 days of in vitro culture, embryos were scored according to Holte et al. [14], and 1-2 embryos were transferred in utero using a Guardia TM Access catheter (Cook Medical ${ }^{\circledR}$, Australia) under TV-US guidance, as previously described [15]. Luteal phase supplementation was achieved by administering $600 \mathrm{mg}$ /day vaginal P (Progeffik®, Effik, Belgium) for 14 days, starting the day of ET. Pregnancy was assessed by serum hCG measurement 15 days after ET, and then confirmed 2 weeks later if at least one gestational sac was visualized at TV-US.

\section{Microbiota analysis}

Patients underwent vaginal and endometrial microbiota analysis at two time points: in the cycle preceding COS (pre-COS) 
and in the cycle in which COS, OPU, and ET were performed (post-COS). For the pre-COS microbiota analysis, the patients were asked to self-monitor ovulation every day at the same time (first urine of the day) from day 10 of the cycle using a commercial kit for LH peak detection in the urine (Clearblue ${ }^{\circledR}$ Ovulation Test, Swiss Precision Diagnostics, Switzerland). When the test turned positive, an outpatient appointment was scheduled 3 days later to confirm spontaneous ovulation by measuring circulating E2 and progesterone levels. On the same day, a mock ET and a vaginal swab were performed: a sterile speculum was inserted and a vaginal swab (Copan eNat® COPAN Diagnostics, USA) was taken from the posterior fornix. Immediately thereafter, ET was simulated using a Guardia® Access catheter (Cook Medical $®$, Australia). The catheter consists of two components: an outer guide and an inner soft catheter; first, the guide is inserted into the cervical canal, avoiding contact with the vaginal walls; the inner soft catheter is then gently inserted into the guide and advanced until reaching the endometrium inside the uterine cavity. The inner catheter is protected from contact with the vagina or the cervix by the outer guide. After retracting the catheter without exposing its inner part to the vaginal environment, the distal end of the inner part (approximately 5-10 mm) was cut using sterile scissors and immediately placed in sterile PCR tubes. To maintain the stability of bacterial DNA [16], vaginal and endometrial samples were frozen at $-80^{\circ} \mathrm{C}$ until analysis. An ultrasound examination was performed at the end of the procedure to check for the presence of corpus luteum.

Post-COS microbiota analysis was performed in exactly the same way at the time of fresh ET. A vaginal swab from the posterior fornix was taken; then, ET was performed as described, with the only difference that a medium droplet containing 1-2 embryos was released about $1 \mathrm{~cm}$ from the uterine fundus under TV-US guidance before retracting the catheter, cutting the tip, and storing it.

\section{5 ribosomal hypervariable region sequencing}

Vaginal swabs and catheter tips underwent specific treatment to obtain equal volumes for DNA extraction: $8 \mu$ of sterile deionized water was added to the catheter tip and $100 \mu$ to the swab. The samples were then gently vortexed to release the bacteria into the solution. The first extraction step involved digestion of the bacterial cell wall by adding 1 (catheter) or 2 (swab) $\mu$ l of lysis buffer (200 mM KOH, $50 \mathrm{mM}$ DTT). After centrifugation, the solution was incubated at $65^{\circ} \mathrm{C}$ for $10 \mathrm{~min}$; 1 (catheter) or 2 (swab) $\mu$ l of neutralizing buffer $(0.9 \mathrm{M}$ Tris$\mathrm{HCl}, \mathrm{pH} 8.3,0.3 \mathrm{M} \mathrm{KCl}, 0.2 \mathrm{M} \mathrm{HCl}$ ) was then added. The extracted DNA was then purified: $10 \mu$ of extract from the swab and the solution from the catheter were recovered, and $20 \mu \mathrm{l}$ of sterile deionized water and $54 \mu \mathrm{l}$ of magnetic beads (Agencourt ${ }^{\circledR}$ AMPure XP reagent, Beckman Coulter, Thermo Fisher Scientific, USA) were added. The samples were then incubated at room temperature for $5 \mathrm{~min}$ to allow the magnetic beads to bind DNA and were then placed in a DynaMag® (Thermo Fisher Scientific, USA) and washed with $300 \mu \mathrm{l}$ of $70 \%$ ethanol to eliminate the supernatant. Both steps were repeated twice. A volume of $15 \mu \mathrm{l}$ sterile deionized water was added to recover the extracted and purified DNA; the pellet obtained after washing with ethanol was re-suspended, and the samples were inserted again in the DynaMag®; the supernatant was recovered, purified, and stored at $-80{ }^{\circ} \mathrm{C}$ until sequencing.

Next-generation sequencing (NGS) of the bacteria-specific $16 \mathrm{~S}$ ribosome gene was performed utilizing a microbiota solution B kit-hypervariable regions V3-V4-V6 (Arrow Diagnostics S.r.1., Italy). The B kit was composed of Enzyme Mix 1 solution containing the enzyme mixture for the PCR target, Enzyme Mix 2 solution containing the enzyme mixture for the PCR index, Amp Mix V3-V6 solution of degenerated oligonucleotides for amplifying hypervariable regions V3-V4-V6 of the bacterial 16S rDNA gene, and oligonucleotide solution for indexing amplified samples with the PCR target (Index Mix). An Illumina ${ }^{\circledR}$ MiSeq $^{\text {TM }}$ system platform (Illumina Inc., USA) was used for sequencing. Raw sequencing data have been uploaded to NCBI, project title: BioProject PRJNA634237.

Taxonomic assignment and bioinformatic analysis were performed using the MicrobAT® software (Microbiota Analysis Tool; SmartSeq S.r.l., Italy). In the first phase of the analysis, reads were cleaned by a dedicated algorithm to remove short, low-quality sequences. Taxonomic assignment was then made by aligning the remaining sequences with the reference database (RDP database release 11-update 5) [17]. Only sequences that met reference criteria in the alignment phase were associated by the analysis system to the species taxonomic level (minimum length of the sequence aligning with the reference sequence $\geq 80 \%$, similarity percentage $\geq$ $97 \%$ ). The results were filtered by elimination of contaminating genera (Sphingomonas, Renibacterium, and Arthrobacter, see Table 4), which were then detected by further analysis performed using devices not in contact with biological material.

Finally, the samples were characterized according to the Shannon diversity index (SDI), and Bray-Curtis analysis of the phylogenetic distance among genera was performed preand post-COS for the vaginal and the endometrial sites.

\section{Statistics}

Statistical analysis was carried out with the R software $\AA$ version 3.4.2 (R Studio, USA). This version was extended with the RAM package, designed for microbial ecology studies, genomic, and metagenomic analysis; it is similar and equivalent to others (Vegan®, Ggplot ${ }^{\circledR}, \operatorname{Labdsv}{ }^{\circledR}$, RColorBrewer®, and Heatplus®; https://www.rstudio.com). 
Quantitative variables were compared using the Wilcoxon signed-rank test. Bray-Curtis distances were compared by non-parametric analysis of similarities (ANOSIM). A $p$ value was derived based on the likelihood ratio test statistic for each factor of interest. Significance was set at $p<0.05$.

\section{Results}

Table 1 presents the patients' baseline characteristics; all women were spontaneously ovulating and had normal P levels in the luteal phase of the cycle preceding COS.

Table 2 presents the outcome of the IVF cycle; the circulating levels of E2 and P at ET (post-COS) were significantly higher than the pre-COS levels $(819.2 \pm 187$ vs. $135.3 \pm$ $41.2 \mathrm{pg} / \mathrm{ml}$ for E2, post-COS, and pre-COS, respectively, $p<0.05 ; 32.1 \pm 7.4$ vs. $11.5 \pm 6.4 \mathrm{ng} / \mathrm{ml}$ for $\mathrm{P}$, post-COS, and pre-COS, respectively, $p<0.05$ ) (Tables 1 and 2).

A total of 81 and 90 bacterial genera were isolated from the vagina and the endometrium, respectively. Figure 1 shows the 30 most prevalent genera of the vaginal and the endometrial microbiota: In $13 / 15$ patients (86.7\%), a higher than $10 \%$ difference in the pre-COS and post-COS bacterial composition of the microbiota (relevant for optimal taxonomic unit (OTU) classification and relative abundance analysis) was detected. The pre-COS vs. post-COS difference was very marked in some samples because of the post-COS increase in potentially pathogenic genera, including Atopobium, Gardnerella, and Pelomonas.

Table 3 lists the ten most prevalent bacterial genera (relative abundance analysis) in the vagina and the endometrium. In the vaginal specimens, Lactobacillus was the most prevalent genus pre-COS and post-COS, although it was less abundant in the post-COS vaginal swabs than before $\operatorname{COS}(71.5 \pm$ $40.6 \%$ pre-COS vs. $61.1 \pm 44.2 \%$ post-COS, respectively;

Table 1 Baseline characteristics of patients. Values are expressed as mean $\pm \mathrm{SD}$

\begin{tabular}{ll}
\hline Patient age (years) & $35.1 \pm 4.3$ \\
Partner age (years) & $39.6 \pm 5.4$ \\
Duration of infertility (years) & $3.3 \pm 1.0$ \\
BMI (kg/m²) & $23.5 \pm 4.4$ \\
Antral follicle count (AFC) & $15.0 \pm 4.3$ \\
AMH (ng/ml) & $3.9 \pm 2.4$ \\
Basal (day 3) FSH (IU/l) & $6.1 \pm 1.3$ \\
Estradiol (LH peak + 3 days, pre-COS; pg/ml) & $135.3 \pm 41.2$ \\
Progesterone (LH peak +3 days, pre-COS; ng/ml) & $11.5 \pm 6.4$ \\
Sperm concentration (M/ml) & $41.4 \pm 25.7$ \\
Sperm progressive (A+B) motility (\%) & $26.0 \pm 13.5$ \\
Sperm normal morphology (\%) & $10.1 \pm 7.1$ \\
\hline
\end{tabular}

$B M I$ body mass index; $A M H$ anti-müllerian hormone; $F S H$ folliclestimulating hormone
Table 2 IVF treatment outcome. Values are expressed as mean $\pm \mathrm{SD}$ or percentage

\begin{tabular}{ll}
\hline Daily exogenous FSH dose (IU) $^{\mathrm{a}}$ & $199.1 \pm 43$ \\
Total exogenous FSH dose (IU) $^{\mathrm{a}}$ & $2467.2 \pm 731$ \\
Peak estradiol at trigger (pg/ml) & $2218.0 \pm 1143$ \\
Progesterone at trigger (ng/ml) & $0.7 \pm 0.8$ \\
Retrieved oocytes & $9.4 \pm 5.5$ \\
Mature (MII) oocytes & $8.0 \pm 4.6$ \\
Fertilized (2PN) oocytes & $5.7 \pm 4.1$ \\
Cleaving embryos & $5.6 \pm 3.9$ \\
Mean embryo score & $7.6 \pm 1.0$ \\
Number of embryos transferred & $1.4 \pm 0.9$ \\
Endometrial thickness at ET (mm) & a \\
Estradiol at ET (pg/ml) & $9.7 \pm 1.7$ \\
Progesterone at ET (ng/ml) & $819.2 \pm 187$ \\
Pregnancy rate/ET & $32.1 \pm 7.4$ \\
\hline
\end{tabular}

${ }^{\text {a }}$ FSH follicle-stimulating hormone; $E T$ embryo transfer

${ }^{\mathrm{b}}$ Embryo score is expressed using the score by Holte et al., 2007

$p>0.05)$. An increase in pathogenic genera in the vaginal microbiota was noted for Prevotella $(3.5 \pm 8.9 \%$ pre-COS vs. $12.0 \pm 19.4 \%$ post-COS, respectively; $p>0.05)$ and Escherichia-Shigella $(1.4 \pm 5.6 \%$ pre-COS vs. $2.0 \pm 7.8 \%$ post-COS, respectively; $p>0.05$ ) (Table 3 ).

The endometrial microbiota was extremely heterogeneous, though Lactobacillus was the prevalent genus pre-COS and remained the prevalent genus post-COS $(27.4 \pm 34.5 \%$ and $25.0 \pm 29.9 \%$, pre-COS and post-COS, respectively; $p>0.05$ ). An overall increase in the prevalence of potentially pathogenic genera was observed post-COS: Prevotella was significantly increased from $3.4 \pm 9.5 \%$ to $4.7 \pm 7.4 \%$ ( $p=$ $0.0494)$, and Atopobium was significantly increased from $0.7 \pm 1.6 \%$ to $5.8 \pm 12.0 \%$ post-COS $(p=0.0178)$.

Fig. 1 Relative abundance of the 30 most present bacterial genera in the vaginal and endometrial microbiota (pre-COS and post-COS). In each line, pre refers to pre-COS analysis, post to post-COS analysis. Other bacteria, not listed in the figure include Finegoldia, Curvibacter, Ochrobactrum, Mobiluncus, Howardella, Bacteroides, Streptophyta, Peptoniphilus, Ruminococcus, Alloscardovia, Paracoccus, Anaerosphaera, Caulobacter, Kocuria, Serratia, Neisseria, Ralstonia, Parvimonas, Solobacterium, Enhydrobacter, Acinetobacter, Enterococcus, Rothia, Granulicatella, Corynebacterium, Actinomyces, Campylobacter, Fusobacterium, Bacillus, Slackia, Peptococcus, Acidaminococcus, Oscillibacter, Saccharibacteria_genera_incertae sedis, Sutterella, Gp3, Cloacibacterium, Moryella, Gemmiger, Spartobacteria genera incertae sedis, Paenibacillus, Negativicoccus, Propionimicrobium, Blautia, Faecalibacterium, Pedobacter, Rhodococcus, Meiothermus, Allisonella, Carnobacterium, Lachnospiracea_incertae sedis, Neochlamydia, Diaphorobacter, Brevibacterium, Methylobacterium, Haemophilus, Micrococcus, Azomonas, Clostridium XVIII, Herbaspirillum, Oribacterium, Sarcina, Nevskia 
Microbial Taxa Abundance

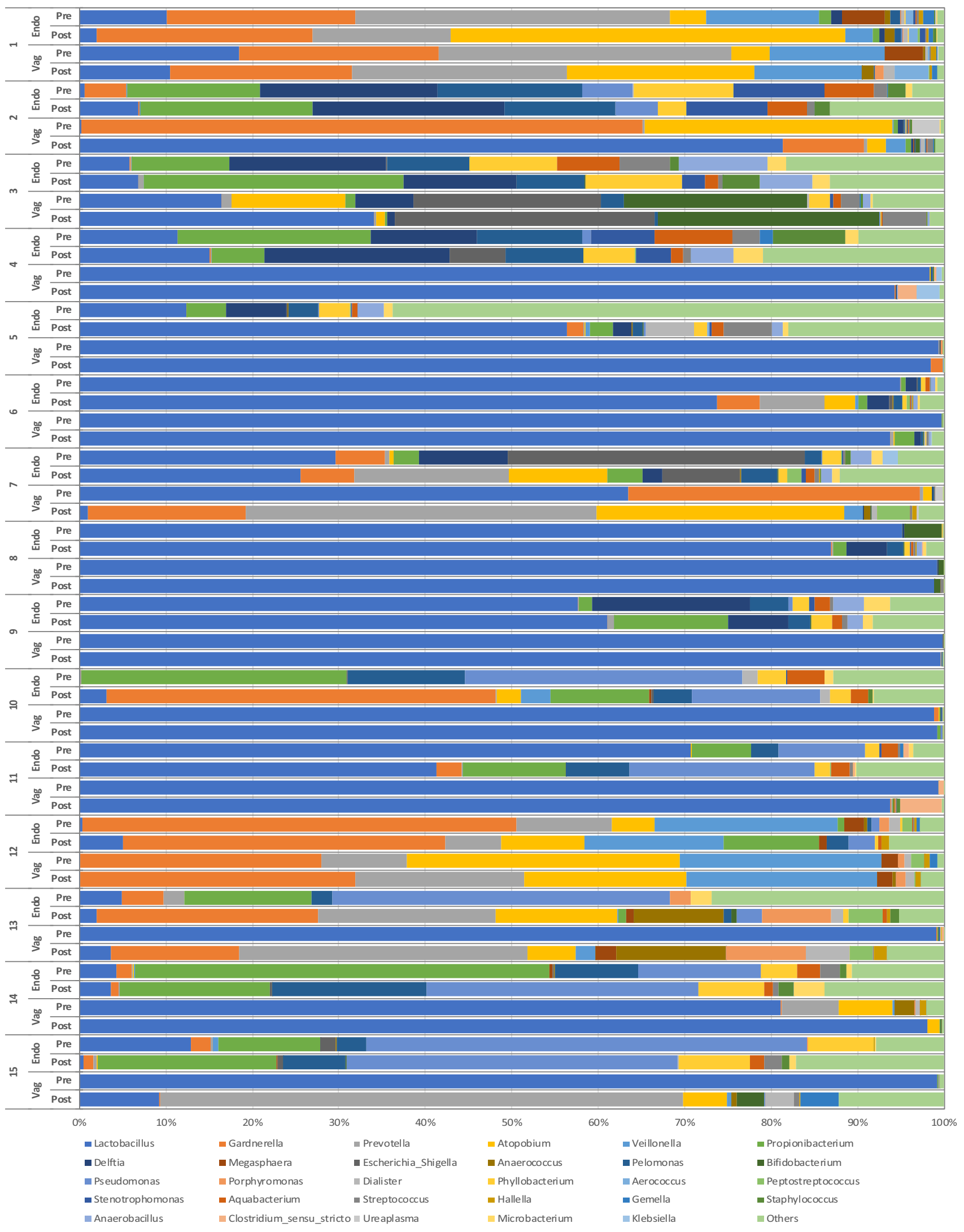


Table 3 Proportion of the 10 most abundant bacterial genera in the vaginal and endometrial microbiota pre-COS and post$\operatorname{COS}$

\begin{tabular}{lllll}
\hline Bacterial genus & $\begin{array}{l}\text { Vagina } \\
\text { pre-COS }\end{array}$ & $\begin{array}{l}\text { Vagina } \\
\text { post-COS }\end{array}$ & $\begin{array}{l}\text { Endometrium } \\
\text { pre-COS }\end{array}$ & $\begin{array}{l}\text { Endometrium } \\
\text { post-COS }\end{array}$ \\
\hline $\begin{array}{l}\text { Lactobacillus } \\
\text { Gardnerella }\end{array}$ & $71.5 \pm 40.6$ & $61.1 \pm 44.2$ & $27.4 \pm 34.5$ & $25.0 \pm 29.9$ \\
Prevotella & $10.0 \pm 19.2$ & $6.5 \pm 10.2$ & $6.1 \pm 13.5$ & $10.1 \pm 15.2$ \\
Propionibacterium & $3.5 \pm 8.9$ & $12.0 \pm 19.4$ & $3.4 \pm 9.5$ & $4.7 \pm 7.4^{*}$ \\
Pseudomonas & $0.1 \pm 0.3$ & $0.3 \pm 0.6$ & $11.5 \pm 13.5$ & $10.2 \pm 8.9$ \\
Atopobium & $0.0 \pm 0.1$ & $0.0 \pm 0.1$ & $10.3 \pm 16.7$ & $7.8 \pm 12.7$ \\
Delftia & $5.7 \pm 10.6$ & $5.6 \pm 9.4$ & $0.7 \pm 1.6$ & $5.8 \pm 12.0^{*}$ \\
Pelomonas & $0.5 \pm 1.7$ & $0.1 \pm 0.3$ & $6.0 \pm 7.9$ & $5.1 \pm 7.7$ \\
Veillonella & $0.2 \pm 0.7$ & $0.1 \pm 0.1$ & $5.5 \pm 5.4$ & $5.4 \pm 5.0$ \\
Escherichia coli/Shigella & $2.5 \pm 6.7$ & $2.8 \pm 6.2$ & $2.3 \pm 6.2$ & $1.6 \pm 4.2$ \\
$\quad$ spp. & $1.4 \pm 5.6$ & $2.0 \pm 7.8$ & $2.5 \pm 8.8$ & $1.1 \pm 2.7$ \\
\hline
\end{tabular}

Values are expressed as percentage $\pm \mathrm{SD}$

${ }^{*} p<0.05$ pre-COS vs. post-COS
A comparison between the most prevalent 20 OTUs in the vaginal and the endometrial samples at pre-COS and postCOS analysis is shown in Fig. 2 a and b, respectively. The slight degree of overlap in the two microbiota (gray part of the columns) suggests a difference in the microbiota at both sampling sites between pre- and post-COS analyses. Also, the Shannon biodiversity index showed differences between preCOS and post-COS genera at both sampling sites (Fig. 3). Biodiversity was greater in the endometrial microbiota at both time points, indicating that vaginal and endometrial microbiota had different characteristics $(p<0.05)$. In both sites, biodiversity was significantly greater after $\operatorname{COS}(p<0.05)$, particularly in the endometrial microbiota, as confirmed by BrayCurtis analysis of the phylogenetic distance among bacteria genera, which resulted significantly higher post-COS (Fig. 4 a and b). Bray-Curtis analysis confirmed significant differences also for the paired endometrium-vagina samples at each time point (Fig. $4 \mathrm{c}$ and d).

\section{Discussion}

Menstrual cycle phases and changes in circulating estrogen levels over a woman's lifespan are associated with changes in the composition of vaginal microbiota $[18,19]$, but the effect(s) of exogenous FSH administration, with the consequent supraphysiological increase in E2, as well as P supplementation in the luteal phase, have not been studied to date.

A similar knowledge gap exists for the endometrial microbiota. Until a few years ago, the uterine cavity was believed to be sterile, and the presence of bacteria was associated with pathological colonization by vaginal bacteria; rather recently, it was demonstrated that the endometrium has its own microbiota and that the microbiological characteristics of the vagina and the endometrium only partially overlap [8].
As ovarian steroids produced during the physiological menstrual cycle affect vaginal microbiota, it seems plausible that also the uterine microbiota could be influenced by the fluctuations in E2 and P levels during cycle phases; this hypothesis has never been investigated using advanced techniques, however. Similarly, the impact of COS and P supplementation during IVF cycles on vaginal and endometrial microbiota has never been studied. Theoretically, the effects of ovarian steroids on the composition of vaginal and endometrial microbiota could be significantly amplified during COS in comparison with the physiological menstrual cycle, as $\mathrm{COS}$ induces wider and more rapid changes in E2 serum levels than the natural cycle, while P supplementation after ET increases circulating P far beyond physiological levels.

Based on these premises, the aim of the present study was to evaluate the impact of COS and P supplementation on vaginal and endometrial microbiota. The latter was sampled using a previously described technique that avoids contamination by vaginal flora [20]. Moreover, the vaginal and the endometrial samples were submitted to a highly sensitive, novel molecular methods (such as NGS of bacterial 16S RNA) for a thorough analysis of the bacterial genera, biodiversity, and microbial instability. Unfortunately, the high costs of the NGS technique limited the possibility of enrolling a larger patient population and to relate microbiota composition to IVF outcome, an endpoint that was beyond the scope of this study.

We report for the first time data suggesting that COS and $\mathrm{P}$ supplementation induce significant changes in the vaginal and the endometrial microbiota at the time of the so-called implantation window $[21,22]$ when fresh ET is performed. The relative proportion of vaginal and endometrial Lactobacillus was decreased during the IVF cycle 
Fig. 2 Distribution of bacterial genera in endometrial (blue part of each column), vaginal (orange part of each column), and endometrial and vaginal (gray part of each column) microbiota, pre-COS (a) and post-COS (b).

Values are expressed as mean number of reads a
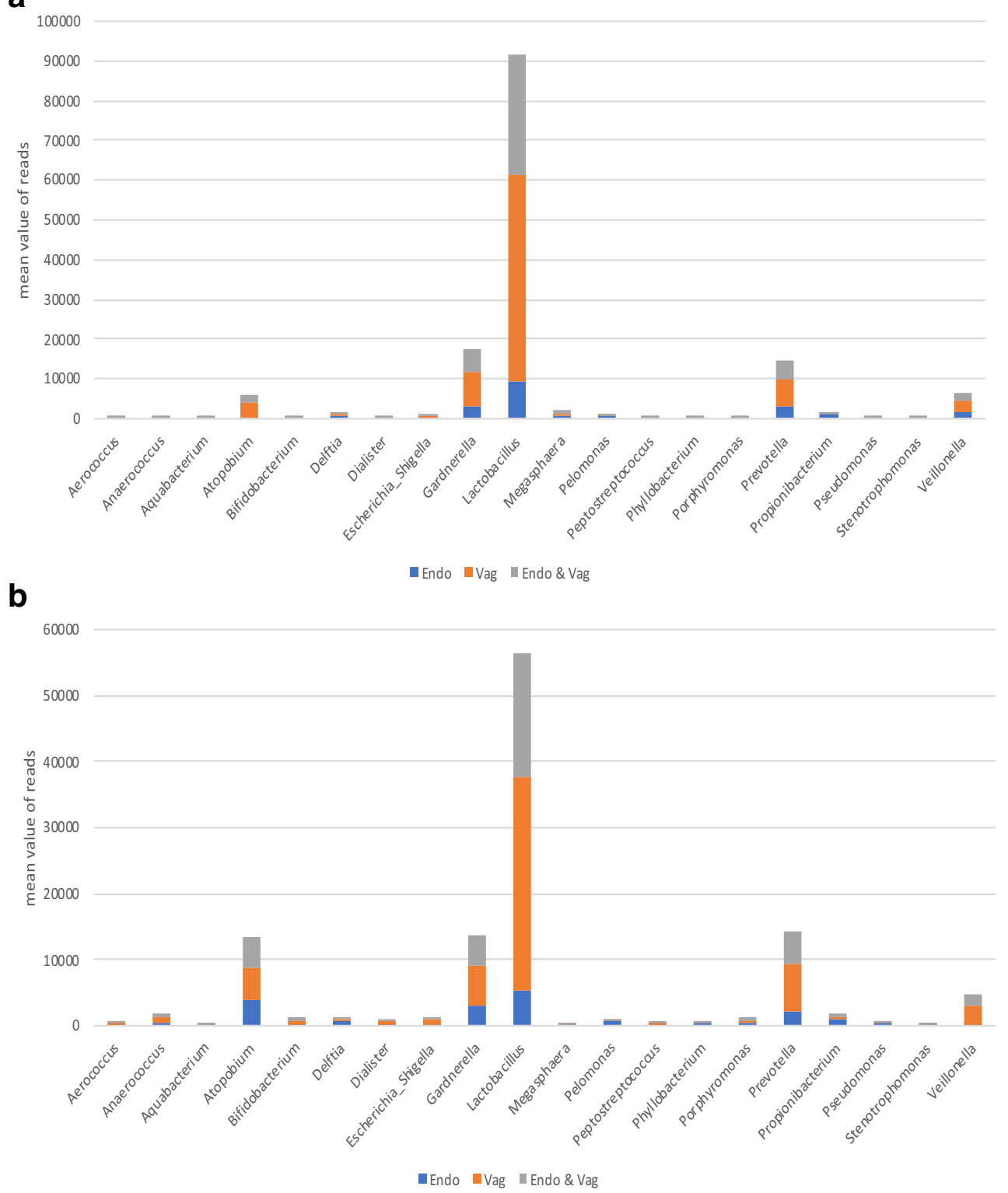

(post-COS), with a simultaneous increase in potentially pathogenic bacteria, such as Atopobium, EscherichiaShigella, and Prevotella. An unfavorable change in the vaginal and the endometrial microbiota after IVF (COS and $\mathrm{P}$ supplementation) occurred: the increase in the microbial instability of the uterine cavity (higher Shannon biodiversity index) was particularly pronounced.

This finding seems to contradict evidence that raising the levels of circulating estrogen induces an increase in Lactobacillus in the vagina [23, 24]. However, the estrogen levels during ART treatment exceed by an order of magnitude the natural levels during spontaneous ovulation, with completely different kinetics depending on the type of COS. The effects of these supraphysiological levels on vaginal and endometrial microbiota have never been investigated so far.
However, whether or not these changes in the endometrial microbiota might affect IVF outcome or increase the incidence of obstetric complications is still unknown. Some authors have criticized the hypothesis that endometrial microbiota plays a role in IVF outcome [25] and a recently published review [26] even questioned the existence of an active endometrial microbiota. The conclusions of the review have been challenged and critical issues raised [27], particularly as regards the variations in endometrial microbiota composition in relation to hormonal changes and compared with the vaginal microbiota. The present study provides evidence in this direction: the Shannon biodiversity index of the vaginal and the endometrial samples differed before and after COS and the biodiversity of the endometrial microbiota appeared to be greater than the vaginal microbiota. In interpreting our results, 
Shannon Value

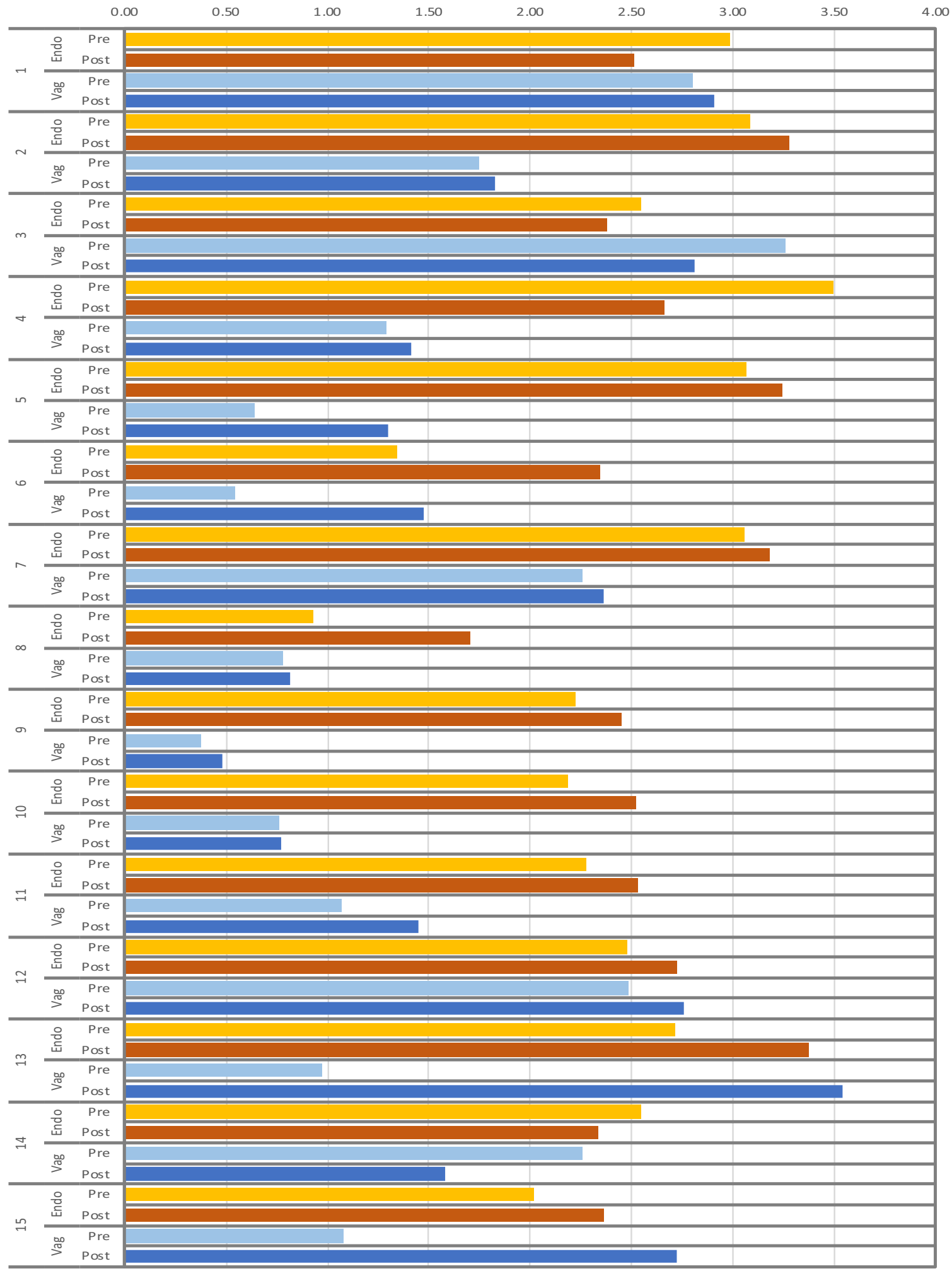

Fig. 3 Shannon values of vaginal and endometrial microbiota, pre-COS and post-COS

a potential contamination effect of the pre-COS on the postCOS analysis should be considered. However, the invasiveness of a vaginal swab and a transfer simulation is negligible, so much so that it is routinely performed by some IVF centers. Furthermore, a blank analysis of the devices used for the analyses was carried out to exclude possible contaminants and 


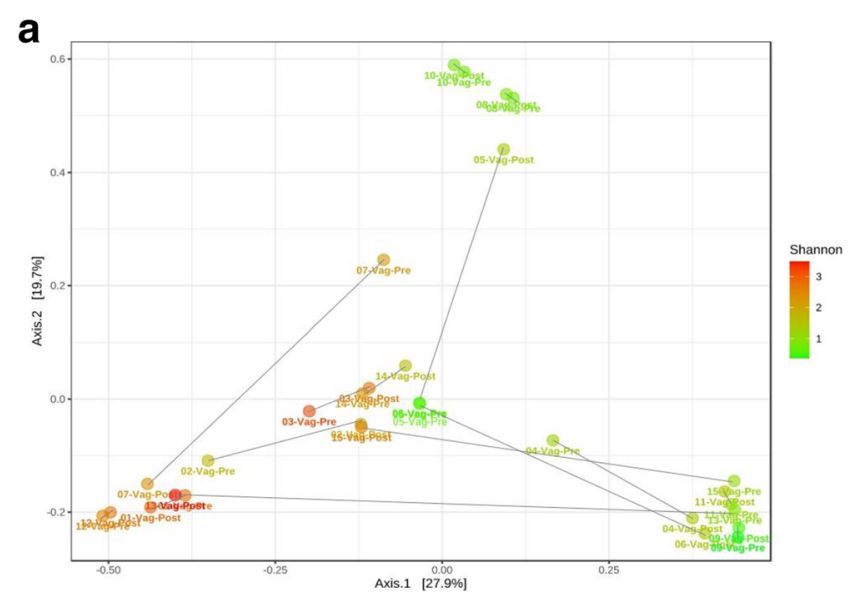

C

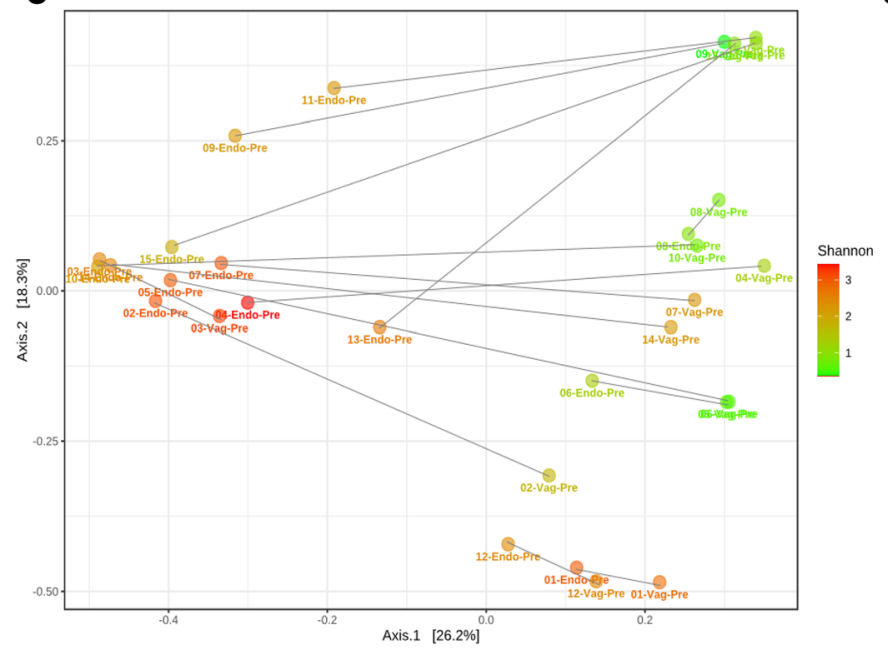

Fig. 4 Analysis of the phylogenetic distance among bacterial genera calculated by the Bray-Curtis method for the endometrium (a) and for the vagina (b) and for the paired each type of sample, pre- and post-COS (c, d). Color data points according to alpha diversity (Shannon index). Connecting lines join the paired samples. The Bray-Curtis distance index

their impact on subsequent analysis. It is unlikely, therefore, that a minimal presence of bacteria, as identified in the blank control (Table 4), could disrupt a microbiota dominated by Lactobacilli in the time (about 1 month) between the two analyses. Another potential contamination could have been the self-administration of vaginal progesterone. Patients were advised to wear sterile gloves during administration and our analyses did not demonstrate a relevant presence of bacteria of cutaneous origin in post-COS results. For these reasons, it is reasonable to assume that the microbiota changes could be COS-related.

Although Lactobacillus was predominant in both, the presence of other bacteria and a more heterogeneous environment compared with the vagina makes the endometrium an interesting niche to explore.

Endometrial dysbiosis and subclinical chronic endometritis have been claimed to be possible causes of repeated implantation failure or recurrent miscarriage [28-30]. Furthermore, b

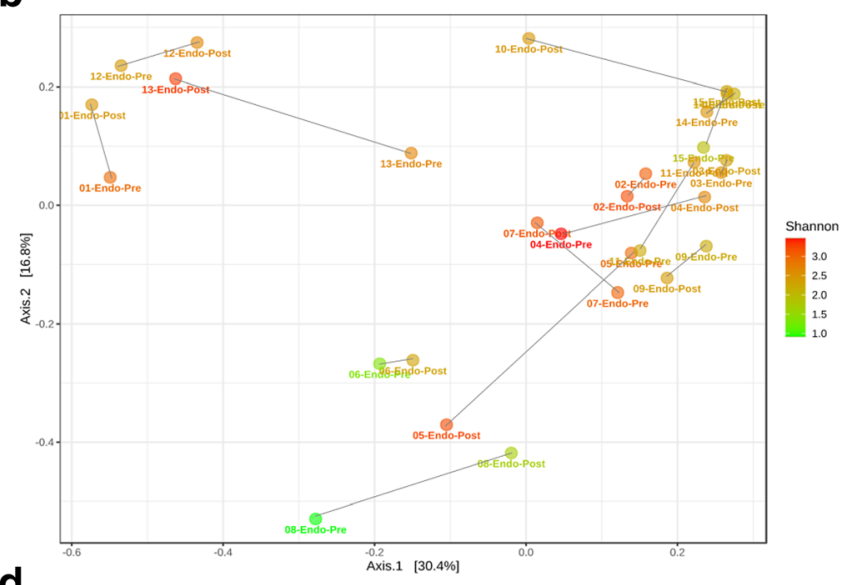

d

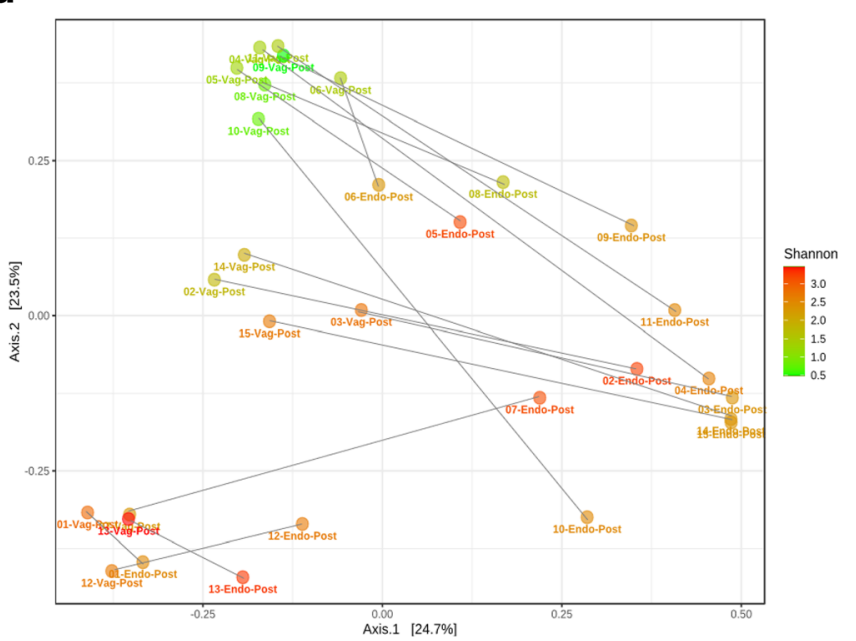

is calculated with the formula $1-(2 w / a+b)$, where $w$ is the sum of the minor score for species that are present in both communities, $a$ is the sum of the taxa measures in a community, and $b$ is the sum of taxa measures in the other community. The distance index was significantly increased post-COS

the relative vaginal abundance of Atopobium, EscherichiaShigella, and Prevotella was associated with preterm delivery [31] and a local pro-inflammatory state potentially affecting placentation [32]. Also, IVF pregnancies are known to be associated with a higher-than-normal risk of obstetrical complications, including those of infectious origin (e.g., preterm delivery, premature rupture of membranes) [33, 34].

Clarifying whether the endometrial microbiota after COS might affect IVF outcome or not will be the objective of our next study, taking into account the reportedly higher implantation rate observed by some authors when, after the so-called freeze-all strategy, thawed embryos are transferred in a cycle different from the one in which COS is performed [35]. Indeed, in a natural cycle, when E2 and P levels are physiological, the reported iatrogenic changes in pre-COS endometrial microbiota would not occur, and embryos could be transferred in a more physiological intrauterine environment. 
Table 4 Analysis of potential contaminants in the "white" sample and the average of the relative abundances detected in vaginal and endometrial analyses. Sphingomonas was excluded from vaginal and endometrial microbiota analysis because recognized by previous works as a contaminant ${ }^{\mathrm{a}}$. Arthrobacter and Renibacterium are genera found in soil and water; we recognized them as contaminants ${ }^{\mathrm{b}, \mathrm{c}}$. The other genera were not excluded because they were present in negligible quantities in the blank sample and described by previous works on vaginal and endometrial microbiota

\begin{tabular}{llll}
\hline \% value & \multicolumn{2}{l}{ Mean \% value } & \multirow{2}{*}{ Genus } \\
\cline { 2 - 3 } Blank control & Endometrial catheter & Vaginal swab \\
\hline 38.54 & 24.36 & 1.00 & Sphingomonas \\
10.65 & 5.32 & 0.28 & Arthrobacter \\
6.38 & 1.71 & 0.10 & Pseudomonas \\
2.04 & 2.17 & 0.08 & Propionibacterium \\
1.44 & 0.05 & 0.00 & Acinetobacter \\
1.26 & 0.21 & 0.03 & Staphylococcus \\
0.43 & 1.08 & 0.03 & Pelomonas \\
0.25 & 0.24 & 0.01 & Renibacterium \\
\hline
\end{tabular}

${ }^{a}$ Hashimoto T, Kyono K. Does dysbiotic endometrium affect blastocyst implantation in IVF patients? J Assist Reprod Genet. 2019;36:2471-9

${ }^{\mathrm{b}}$ Koh H-W, Kang M-S, Lee K-E, Lee E-Y, Kim H, Park S-J. Arthrobacter dokdonellae sp. nov., isolated from a plant of the genus Campanula. J Microbiol. 2019;57:732-7

${ }^{\mathrm{c}}$ Hirvelä-Koski V. Renibacterium salmoninarum: effect of hypochlorite treatment, and survival in water. Dis Aquat Org. 2004;59:27-33

One limitation of the present study is the small study sample size $(n=15)$. Nonetheless, analysis of four samples from the same patient, taken at two sites and at different time points in IVF treatment, makes this study one of the few with a highly detailed characterization of intra-individual variability of the microbiota of the female reproductive tract. This is a fundamental theme in the study of microbiota. Indeed, frequent fluctuations in the composition of vaginal microbiota have been documented by microscopy and cultivation studies [36-38]. Fluctuations can be triggered by genetic predisposition in different ethnic groups, menstruation or sexual behaviors, and a history of bacterial vaginosis or be driven by uncharacterized factors, especially in not Lactobacillusdominated microbiota [39]. Although we have tried to limit these factors (Caucasian ethnicity, exclusion of patients with cervico-vaginal infections in the last 6 months), intra-individual variability in microbiota study remains a factor to be taken into account, and the results should be interpreted with caution.

Further research into the impact of the microbiota on reproduction should be focused in this direction. For example, it is necessary to integrate information on the female microbiota with that of seminal fluid, which has its own microbiota [40, 41]. Indeed, it is likely that in the future our attention will shift from studying the individual microbiota to the "reproductive microbiota" of the couple. In this view, it will be interesting to integrate the impact of the "bacterial microbiota" with that of the "virota," which together can potentially impact IVF outcomes, as recently demonstrated [42].

Furthermore, it will be of pivotal importance to define the characteristics of the endometrial microbiota and understand its physiological alterations during the menstrual cycle. These premises are fundamental to fully understand the potential repercussions of the microbiota on implantation and also on the evolution of pregnancy. Clinicians must be aware of the fluctuations of the reproductive microbiota in order to identify the transitory conditions that can be associated with better reproductive and obstetric outcomes.

In conclusion, our pilot study shows for the first time with NGS of the $16 \mathrm{~S}$ ribosomal subunit that COS and P supplementation routinely performed during IVF treatment induce unfavorable changes in the composition of vaginal and endometrial microbiota, increasing the proportion of potentially pathogenetic genera at both sites and significantly increasing the biodiversity and the environmental instability of the uterine cavity during the so-called implantation window.

Acknowledgments The authors wish to thank Dr. F. Di Pierro for critically reviewing the article. We thank Sara Cesarano, Nicole Brunod, Sara Leoncini, and Carlotta Scarafia for their assistance in the sample collection and the staff of Sant Anna Hospital, Turin, Italy, and of Arrow Diagnostics, Genoa, Italy.

Authors' contributions A.C. conceived the study design and wrote the article. E.Z. and L.B. significantly contributed to the data analysis, interpreted the data, critically revised the article, and approved the final version. A.R., G.G, and S.C significantly contributed to the execution of the study and drafting of the manuscript, and approved the final version. S.C., F.B., A.T., C.P., C.C., F.S, P.B., and R.C contributed to the execution of the study, critically revised the article, and approved the final version. C.B. contributed to coordinate the study and critically revised the manuscript.

Funding information Open access funding provided by Università degli Studi di Torino within the CRUI-CARE Agreement. This research was funded with resources from the University of Turin. No external funds have been received.

Data availability All data are available under request to the authors.

\section{Compliance with ethical standards}

Conflict of interests The authors declare that they have no conflict of interest.

Ethics approval All procedures performed in studies involving human participants were in accordance with the ethical standards of the institutional and/or national research committee and with the 1964 Helsinki Declaration and its later amendments or comparable ethical standards. The study was approved by the Bioethics Committee of the Medical University of Torino (authorization n. 0092218).

Consent to participate and for publication Informed consent was obtained from all individual participants included in the study. 
Open Access This article is licensed under a Creative Commons Attribution 4.0 International License, which permits use, sharing, adaptation, distribution and reproduction in any medium or format, as long as you give appropriate credit to the original author(s) and the source, provide a link to the Creative Commons licence, and indicate if changes were made. The images or other third party material in this article are included in the article's Creative Commons licence, unless indicated otherwise in a credit line to the material. If material is not included in the article's Creative Commons licence and your intended use is not permitted by statutory regulation or exceeds the permitted use, you will need to obtain permission directly from the copyright holder. To view a copy of this licence, visit http://creativecommons.org/licenses/by/4.0/.

\section{References}

1. Moreno I, Simon C. Deciphering the effect of reproductive tract microbiota on human reproduction. Reprod Med Biol. 2019;18: 40-50.

2. Woo PCY, Lau SKP, Teng JLL, Tse H, Yuen K-Y. Then and now: use of 16S rDNA gene sequencing for bacterial identification and discovery of novel bacteria in clinical microbiology laboratories. Clin Microbiol Infect. 2008;14:908-34.

3. Turnbaugh PJ, Ley RE, Hamady M, Fraser-Liggett CM, Knight R, Gordon JI. The human microbiome project. Nature. 2007;449:804-10.

4. Ravel J, Gajer P, Abdo Z, Schneider GM, Koenig SSK, McCulle SL, et al. Vaginal microbiome of reproductive-age women. Proc Natl Acad Sci U S A. 2011;108(Suppl 1):4680-7.

5. Sirota I, Zarek SM, Segars JH. Potential influence of the microbiome on infertility and assisted reproductive technology. Semin Reprod Med. 2014;32:35-42.

6. Pelzer ES, Allan JA, Waterhouse MA, Ross T, Beagley KW, Knox CL. Microorganisms within human follicular fluid: effects on IVF. PLoS One. 2013;8:e59062.

7. Franasiak JM, Scott RT. Introduction: microbiome in human reproduction. Fertil Steril. 2015;104:1341-3.

8. Moreno I, Codoñer FM, Vilella F, Valbuena D, Martinez-Blanch JF, Jimenez-Almazán J, et al. Evidence that the endometrial microbiota has an effect on implantation success or failure. Am J Obstet Gynecol. 2016;215:684-703.

9. Kyono K, Hashimoto T, Nagai Y, Sakuraba Y. Analysis of endometrial microbiota by $16 \mathrm{~S}$ ribosomal RNA gene sequencing among infertile patients: a single-center pilot study. Reprod Med Biol. 2018;17:297-306.

10. Hyman RW, Fukushima M, Diamond L, Kumm J, Giudice LC, Davis RW. Microbes on the human vaginal epithelium. Proc Natl Acad Sci U S A. 2005;102:7952-7.

11. Roque M. Freeze-all policy: is it time for that? J Assist Reprod Genet. 2015;32:171-6.

12. Roque M, Valle M, Guimarães F, Sampaio M, Geber S. Freeze-all cycle for all normal responders? J Assist Reprod Genet. 2017;34: 179-85.

13. Zhu Q, Chen Q, Wang L, Lu X, Lyu Q, Wang Y, et al. Live birth rates in the first complete IVF cycle among 20687 women using a freeze-all strategy. Hum Reprod. 2018;33:924-9.

14. Holte J, Berglund L, Milton K, Garello C, Gennarelli G, Revelli A, et al. Construction of an evidence-based integrated morphology cleavage embryo score for implantation potential of embryos scored and transferred on day 2 after oocyte retrieval. Hum Reprod. 2007;22:548-57.

15. Revelli A, Rovei V, Dalmasso P, Gennarelli G, Racca C, Evangelista $\mathrm{F}$, et al. Large randomized trial comparing transabdominal ultrasound-guided embryo transfer with a technique based on uterine length measurement before embryo transfer. Ultrasound Obstet Gynecol. 2016;48:289-95.

16. Shabihkhani M, Lucey GM, Wei B, Mareninov S, Lou JJ, Vinters $\mathrm{HV}$, et al. The procurement, storage, and quality assurance of frozen blood and tissue biospecimens in pathology, biorepository, and biobank settings. Clin Biochem. 2014;47:258-66.

17. Cole JR, Wang Q, Fish JA, Chai B, McGarrell DM, Sun Y, et al. Ribosomal Database Project: data and tools for high throughput rRNA analysis. Nucleic Acids Res. 2014;42:D633-42.

18. Muhleisen AL, Herbst-Kralovetz MM. Menopause and the vaginal microbiome. Maturitas. 2016;91:42-50.

19. Power ML, Quaglieri C, Schulkin J. Reproductive microbiomes: a new thread in the microbial network. Reprod Sci. 2017;24:1482-92.

20. Franasiak JM, Werner MD, Juneau CR, Tao X, Landis J, Zhan Y, et al. Endometrial microbiome at the time of embryo transfer: nextgeneration sequencing of the $16 \mathrm{~S}$ ribosomal subunit. J Assist Reprod Genet. 2016;33:129-36.

21. Achache H, Revel A. Endometrial receptivity markers, the journey to successful embryo implantation. Hum Reprod Update. 2006;12:731-46.

22. Coughlan $\mathrm{C}$. What to do when good-quality embryos repeatedly fail to implant. Best Pract Res Clin Obstet Gynaecol. 2018;53:48-59.

23. Vitali D, Wessels JM, Kaushic C. Role of sex hormones and the vaginal microbiome in susceptibility and mucosal immunity to HIV-1 in the female genital tract. AIDS Res Ther. 2017;14:39.

24. Shen J, Song N, Williams CJ, Brown CJ, Yan Z, Xu C, et al. Effects of low dose estrogen therapy on the vaginal microbiomes of women with atrophic vaginitis. Sci Rep. 2016;6:24380.

25. Hashimoto T, Kyono K. Does dysbiotic endometrium affect blastocyst implantation in IVF patients? J Assist Reprod Genet. 2019;36:2471-9.

26. Baker JM, Chase DM, Herbst-Kralovetz MM. Uterine microbiota: residents, tourists, or invaders? Front Immunol. 2018;9:208.

27. Altmäe S. Commentary: uterine microbiota: residents, tourists, or invaders? Front Immunol. 2018;9:1874.

28. Kasius JC, Fatemi HM, Bourgain C, Sie-Go DMDS, Eijkemans RJC, Fauser BC, et al. The impact of chronic endometritis on reproductive outcome. Fertil Steril. 2011;96:1451-6.

29. Cicinelli E, Matteo M, Tinelli R, Lepera A, Alfonso R, Indraccolo $U$, et al. Prevalence of chronic endometritis in repeated unexplained implantation failure and the IVF success rate after antibiotic therapy. Hum Reprod. 2015;30:323-30.

30. Bouet P-E, El Hachem H, Monceau E, Gariépy G, Kadoch I-J, Sylvestre C. Chronic endometritis in women with recurrent pregnancy loss and recurrent implantation failure: prevalence and role of office hysteroscopy and immunohistochemistry in diagnosis. Fertil Steril. 2016;105:106-10.

31. Bretelle F, Rozenberg P, Pascal A, Favre R, Bohec C, Loundou A, et al. High Atopobium vaginae and Gardnerella vaginalis vaginal loads are associated with preterm birth. Clin Infect Dis. 2015;60:860-7.

32. Caselli E, Soffritti I, D'Accolti M, Piva I, Greco P, Bonaccorsi G. Atopobium vaginae and Porphyromonas somerae induce proinflammatory cytokines expression in endometrial cells: a possible implication for endometrial cancer? Cancer Manag Res. 2019;11:8571-5.

33. Nadeau HCG, Subramaniam A, Andrews WW. Infection and preterm birth. Semin Fetal Neonatal Med. 2016;21:100-5.

34. Cavoretto P, Candiani M, Giorgione V, Inversetti A, Abu-Saba $\mathrm{MM}$, Tiberio F, et al. Risk of spontaneous preterm birth in singleton pregnancies conceived after IVF/ICSI treatment: meta-analysis of cohort studies. Ultrasound Obstet Gynecol. 2018;51:43-53.

35. Anav M, Phillips S, Ferrieres-Hoa A, Gala A, Fournier A, Vincens C, et al. Cryopreserved embryo replacement is associated with higher birthweight compared with fresh embryo: multicentric sibling embryo cohort study. Sci Rep. 2019;9:13402.

36. Hay PE, Morgan DJ, Ison CA, Bhide SA, Romney M, McKenzie P, et al. A longitudinal study of bacterial vaginosis during pregnancy. Br J Obstet Gynaecol. 1994;101:1048-53. 
37. Keane FE, Ison CA, Taylor-Robinson D. A longitudinal study of the vaginal flora over a menstrual cycle. Int J STD AIDS. 1997;8: 489-94.

38. Schwebke JR, Richey CM, Weiss HL. Correlation of behaviors with microbiological changes in vaginal flora. J Infect Dis. 1999;180:1632-6.

39. Gajer P, Brotman RM, Bai G, Sakamoto J, Schütte UME, Zhong X, et al. Temporal dynamics of the human vaginal microbiota. Sci Transl Med. 2012;4:132ra52.

40. Baud D, Pattaroni C, Vulliemoz N, Castella V, Marsland BJ, Stojanov M. Sperm microbiota and its impact on semen parameters. Front Microbiol. 2019;10:234.
41. Hou D, Zhou X, Zhong X, Settles ML, Herring J, Wang L, et al. Microbiota of the seminal fluid from healthy and infertile men. Fertil Steril. 2013;100:1261-9.

42. Eskew AM, Stout MJ, Bedrick BS, Riley JK, Omurtag KR, Jimenez PT, et al. Association of the eukaryotic vaginal virome with prophylactic antibiotic exposure and reproductive outcomes in a subfertile population undergoing in vitro fertilisation: a prospective exploratory study. BJOG. 2020;127:208-16.

Publisher's note Springer Nature remains neutral with regard to jurisdictional claims in published maps and institutional affiliations. 